\title{
Canonical quantization of the Gowdy model
}

\author{
Guillermo A. Mena Marugán \\ Instituto de Matemáticas y Física Fundamental, CSIC, Serrano 121, 28006 Madrid, Spain
}

(Received 8 November 1996)

\begin{abstract}
The family of Gowdy universes with the spatial topology of a three-torus is studied both classically and quantum mechanically. Starting with the Ashtekar formulation of Lorentzian general relativity, we introduce a gauge-fixing procedure to remove almost all of the nonphysical degrees of freedom. In this way, we arrive at a reduced model that is subject only to one homogeneous constraint. The phase space of this model is described by means of a canonical set of elementary variables. These are two real, homogeneous variables and the Fourier coefficients for four real fields that are periodic in the angular coordinate which does not correspond to a Killing field of the Gowdy spacetimes. We also obtain the explicit expressions for the line element and reduced Hamiltonian. We then proceed to quantize the system by representing the elementary variables as linear operators acting on a vector space of analytic functionals. The inner product on that space is selected by imposing Lorentzian reality conditions. We find the quantum states annihilated by the operator that represents the homogeneous constraint of the model and construct with them the Hilbert space of physical states. Finally, we derive the general form of the quantum observables of the model. [S0556-2821(97)04514-1]
\end{abstract}

PACS number(s): 04.60.Ds

\section{INTRODUCTION}

The alternative formalism for general relativity put forward by Ashtekar $[1,2]$ has renewed the hopes of consistently quantizing the gravitational interaction in a nonperturbative way. In contrast with the situation found in the geometrodynamic formulation, the gravitational constraints acquire a simple, polynomic form in terms of the Ashtekar canonical variables. In addition, by shifting the emphasis from geometrodynamics to connection dynamics, the introduction of the Ashtekar variables has allowed the use in gravity of mathematical techniques that had been developed in the quantization of gauge field theories.

In order to gain insight into the kind of problems that one will probably have to face when quantizing full general relativity, a lot of attention has been devoted in the last years to the quantization of gravitational models with different types of spacetime symmetries [3,4]. Most of the systems studied are, however, minisuperspace models [3]. These are clearly inadequate to discuss the difficulties that will presumably arise in the quantization of full gravity owing to the presence of an infinite number of degrees of freedom. A possible way to analyze such difficulties would be to consider the quantization of midisuperspace models. The symmetry of this type of models is not large enough as to eliminate all the local degrees of freedom, so that their quantization will lead to a true quantum field theory.

In a recent paper [4], Ashtekar and Pierri carried out the quantization of the Einstein-Rosen cylindrically symmetric spacetimes [5], completing previous works on the subject by Kuchar [6] and Allen [7]. To our knowledge, this is the only gravitational midisuperspace model that has been rigorously quantized in the literature.

It would be of interest to have at our disposal other examples of midisuperspace models whose quantization can be achieved. Natural candidates for such models are provided by spacetimes with two commuting spacelike Killing fields [8]. These spacetimes can generally be described by two lo- cal physical degrees of freedom which depend only on one of the spatial coordinates. In addition, since the pioneering work by Geroch [9], it is known that the Einstein equations of these spacetimes present an infinite number of symmetries. It is, therefore, believed that such systems may in fact be classically integrable, because there should exist a conserved charge associated with each of the symmetries of the Geroch group [10]. Thus, these systems seem to be simple enough as to expect that their quantization may be feasible.

On the other hand, the existence of the Geroch symmetries is on the basis of a series of solution-generating techniques [11] that have been developed from different points of view to obtain new solutions to the Einstein equations. Thanks to these techniques, it has been possible to find a variety of physically interesting classical spacetimes with two commuting Killing fields.

Actually, a particular family of spacetimes of this kind is given by the Einstein-Rosen solutions considered in Ref. [4]. In these solutions, the sections of constant time are noncompact, and the Killing fields are hypersurface orthogonal. In this paper, we will focus our attention on spacetimes which, by contrast, have closed spacelike hypersurfaces and whose commuting spacelike Killing fields are, in general, not orthogonal. The global structure of the spacetimes with these properties has been studied by Gowdy [12], who has shown that, in this case, the sections of constant time must be homeomorphic to either $S^{1} \times S^{2}$ (a three-handle), a threesphere, or a three-torus (or to a manifold covered by one of the above). Among these possible spatial topologies, we will limit our discussion exclusively to the case of a three-torus.

A partial symmetry reduction of this Gowdy model can be found in Ref. [13]. Preliminary studies of its quantization, assuming the orthogonality of the two Killing fields, have been carried out by Berger [14]. In addition, Husain [15,16] has recently proposed gauge-fixing conditions in the Ashtekar formulation for removing all the nondynamical degrees of freedom of the model. However, he has not performed the gauge fixing to completion. On the other hand, Husain has 
not addressed the quantization of the system in Refs. $[15,16]$. Our purpose here is to complete the gauge-fixing procedure and construct a quantum framework for the description of the family of Gowdy cosmologies with the spatial topology of a three-torus.

The paper is organized as follows. Section II deals with the two commuting spacelike Killing field reduction of the Ashtekar formalism for the case of the Gowdy cosmologies. In that section, we also present our model and display the expressions of the first-class constraints. In Sec. III we introduce a set of gauge-fixing conditions and show that they are well posed and consistent. The final result of our gauge fixing is that we can remove all the first-class constraints, except for a homogeneous one. This homogeneous constraint is analogous to the periodicity condition discovered by Gowdy [12]. The classical reduced model determined by our gaugefixing conditions is studied in Sec. IV. We prove that the phase space of the model can be described by using a canonical set of elementary variables that are all real. These are given by four functions on $S^{1}$ and two homogeneous variables. In addition, we explicitly obtain the metric and the reduced Hamiltonian that generates the dynamical evolution. Section V is devoted to the quantization of the above reduced model following the canonical quantization program elaborated by Ashtekar [2]. We first choose a representation space for the quantum theory and select an inner product on it by imposing reality conditions $[2,17]$. The homogeneous constraint of the system is then imposed in the manner of Dirac. The kernel of the quantum constraint provides us with the Hilbert space of physical states. In Sec. VI, we determine the form of the quantum observables of the reduced model and discuss the quantum evolution. Finally, Sec. VII contains the conclusions and some further comments.

\section{THE GOWDY MODEL}

The Gowdy universes are four-dimensional vacuum spacetimes with compact spacelike hypersurfaces and two commuting spacelike Killing fields [12]. In this paper, we are going to analyze only the case in which the spatial topology is that of a three-torus. In addition, we will restrict our considerations to nondegenerate Lorentzian metrics.

Let us first introduce the Ashtekar formalism for Lorentzian general relativity, particularizing then to the Gowdy model. The Ashtekar gravitational variables can be taken as a densitized triad $\widetilde{E}_{i}^{a}$ and a $\mathrm{SO}(3)$ connection $A_{a}^{i}$, both defined on a three-manifold $\Sigma$ [2]. Lower case Latin letters from the beginning and the middle of the alphabet denote spatial and $\mathrm{SO}(3)$ indices, respectively. The $\mathrm{SO}(3)$ indices run from 1 to 3 , and are raised and lowered with the metric $\eta_{i j}=\operatorname{diag}(1,1,1)$. For Lorentzian gravity, the Poisson bracket structure is given by

$$
\left\{A_{a}^{i}(x), \widetilde{E}_{j}^{b}(y)\right\}=i \delta_{a}^{b} \delta_{j}^{i} \delta^{(3)}(x-y) .
$$

In this formula, $x$ and $y$ are two generic points of $\Sigma, \delta_{a}^{b}$ is the Kronecker $\delta$, and $\delta^{(3)}$ is the $\delta$ function on $\Sigma$.

Provided that the metric is nondegenerate, the Ashtekar variables can be expressed in terms of the triad $e_{i}^{a}$ and the extrinsic curvature [18] $k_{a b}$,

$$
\widetilde{E}_{i}^{a}=e_{i}^{a} h(e), \quad A_{a}^{i}=\Gamma_{a}^{i}(e)-i k_{a b} e^{b i},
$$

where $h=\left(\operatorname{det} h_{a b}\right)^{1 / 2}, h^{a b}=e_{i}^{a} e^{b i}$ is the inverse threemetric, and $\Gamma_{a}^{i}$ is the $\mathrm{SO}(3)$ connection compatible with the triad [19],

$$
\Gamma_{a}^{i}=-\frac{1}{2} \epsilon_{\sim}^{i j k}{\underset{\sim}{j b}}_{j b}\left(\partial_{a} \widetilde{E}_{k}^{b}+\Gamma_{c a}^{b} \widetilde{E}_{k}^{c}\right) .
$$

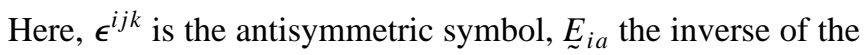
densitized triad, and $\Gamma_{b c}^{a}$ the Christoffel symbols [18].

In the Ashtekar formalism, the first-class constraints of vacuum general relativity are [2]

$$
\begin{gathered}
\mathcal{G}_{i} \equiv \mathcal{D}_{a} \widetilde{E}_{i}^{a}=\partial_{a} \widetilde{E}_{i}^{a}+\epsilon_{i j}{ }^{k} A_{a}^{j} \widetilde{E}_{k}^{a}=0, \\
\mathcal{C}_{a} \equiv F_{a b}^{i} \widetilde{E}_{i}^{b}=0, \\
\mathcal{H} \equiv \epsilon_{i}{ }^{j k} F_{a b}^{i} \widetilde{E}_{j}^{a} \widetilde{E}_{k}^{b}=0,
\end{gathered}
$$

where $F_{a b}^{i}$ is the curvature of the $\mathrm{SO}(3)$ connection,

$$
F_{a b}^{i}=\partial_{a} A_{b}^{i}-\partial_{b} A_{a}^{i}+\epsilon_{j k}^{i} A_{a}^{j} A_{b}^{k} .
$$

For the Gowdy universes with the topology of a threetorus, we can always choose spatial coordinates $\omega, \nu$, and $\theta$ such that $\left(\partial_{\omega}\right)^{a}$ and $\left(\partial_{\nu}\right)^{a}$ are the two commuting Killing fields. For later convenience, we will normalize the periods of these coordinates so that $2 \pi \omega, 2 \pi \nu, \theta \in S^{1}$. All variables of the model must then depend only on $\theta$ and the time coordinate $t$. Furthermore, they have to be periodic in $\theta$ $\in S^{1}$.

On the other hand, following Husain and Smolin [13], we can set equal to zero the densitized triad components

$$
\widetilde{E}_{1}^{\theta}=\widetilde{E}_{2}^{\theta}=\widetilde{E}_{3}^{\omega}=\widetilde{E}_{3}^{\nu}=0 .
$$

The constraints $\mathcal{G}_{1}, \mathcal{G}_{2}, \mathcal{C}_{\omega}$, and $\mathcal{C}_{\nu}$ are then solved by

$$
A_{\theta}^{1}=A_{\theta}^{2}=A_{\omega}^{3}=A_{\nu}^{3}=0 .
$$

After this symmetry reduction, and renaming $A=A_{\theta}^{3}$, $E=\widetilde{E}_{3}^{\theta}$, the remaining first-class constraints of the system can be written [15]

$$
\begin{gathered}
G \equiv \partial_{\theta} E+J=0, \\
C \equiv \widetilde{E}_{L}^{\alpha} \partial_{\theta} A_{\alpha}^{L}+A J=0, \\
H \equiv 2 E \widetilde{E}_{L}^{\alpha} \epsilon^{L}{ }_{M} \partial_{\theta} A_{\alpha}^{M}+2 A E K-K_{\alpha}{ }^{\beta} K_{\beta}{ }^{\alpha}+K^{2}=0,
\end{gathered}
$$

where $\alpha, \beta=\omega$ or $\nu, L, M=1$ or $2, \epsilon^{L M}$ is the antisymmetric symbol in two dimensions, and we have employed the notation

$$
\begin{gathered}
K_{\alpha}{ }^{\beta}=A_{\alpha}^{I} \widetilde{E}_{I}^{\beta}, \quad K=K_{\alpha}{ }^{\alpha}, \\
J_{\alpha}{ }^{\beta}=\epsilon_{L}{ }^{M} A_{\alpha}^{L} \widetilde{E}_{M}^{\beta}, \quad J=J_{\alpha}{ }^{\alpha} .
\end{gathered}
$$


Thus, our Gowdy model can be described by the ten fields $\left(A, E, A_{\alpha}^{L}, \widetilde{E}_{L}^{\alpha}\right)$, which are periodic functions of $\theta$. The Lorentzian symplectic structure is determined by the Poisson brackets

$$
\begin{gathered}
\left\{A(\theta), E\left(\theta^{\prime}\right)\right\}=i \delta\left(\theta-\theta^{\prime}\right), \\
\left\{A_{\alpha}^{L}(\theta), \widetilde{E}_{M}^{\beta}\left(\theta^{\prime}\right)\right\}=i \delta_{\alpha}^{\beta} \delta_{M}^{L} \delta\left(\theta-\theta^{\prime}\right),
\end{gathered}
$$

$\delta(\theta)$ being the $\delta$ function on $S^{1}$. These fields are subject to the constraints $(2.10)-(2.12)$, which will be referred from now on as the Gauss, diffeomorphism, and scalar constraint, respectively. Their physical interpretation and Poisson algebra has been discussed by Husain $[15,16]$.

It is worth noting that the variables $K_{\alpha}{ }^{\beta}$ and $J_{\alpha}{ }^{\beta}$ are not functionally independent, for one can check that $\operatorname{det} K_{\alpha}{ }^{\beta}=\operatorname{det} J_{\alpha}{ }^{\beta}$. Therefore, one cannot replace the elementary variables $\left(A_{\alpha}^{L}, \widetilde{E}_{L}^{\alpha}\right)$ with the eight Gauss-invariant quantities $\left(K_{\alpha}{ }^{\beta}, J_{\alpha}{ }^{\beta}\right)$.

It will prove most convenient to introduce instead a change of phase space variables from $\left(A_{\alpha}^{L}, \widetilde{E}_{L}^{\alpha}\right)$ to $K_{\omega}{ }^{\omega}$, $K_{\omega}^{\nu}, K, J$, and

$$
\begin{gathered}
x=\frac{q^{\omega \omega}}{q^{\nu \nu}}, \quad v=\frac{q^{\omega \nu}}{q^{\nu \nu}}, \\
w=\frac{1}{2} \ln q^{\nu \nu}, \quad \phi=\arctan \left(\frac{\widetilde{E}_{1}^{\nu}}{\widetilde{E}_{2}^{\nu}}\right),
\end{gathered}
$$

where

$$
q^{\alpha \beta}=\widetilde{E}_{L}^{\alpha} \widetilde{E}^{\beta L}
$$

From Eqs. (2.2) and (2.8), we get that $q^{\alpha \beta}=h^{\alpha \beta} h^{2}$, so that, for positive definite three-metrics, $q^{\alpha \beta}$ must also be positive definite. Therefore, $x, v$ and $w$ are well defined by Eqs. (2.17) and (2.18), and we must have $x>0, v, w \in \mathbb{R}$, and $x>v^{2}$, this last inequality coming from the fact that

$$
\operatorname{det} q^{\alpha \beta}=e^{4 w}\left(x-v^{2}\right)>0 .
$$

As to the variables $K_{\omega}{ }^{\omega}, K_{\omega}{ }^{\nu}, K, J$, and $\phi$, we will admit for the moment that they are complex.

Let us show that the above change of variables can always be inverted in the sector of positive definite three-metrics. Using Eq. (2.19), relations (2.18) can be equivalently written in the form

$$
\widetilde{E}_{1}^{\nu}=e^{w} \sin \phi, \quad \widetilde{E}_{2}^{\nu}=e^{w} \cos \phi .
$$

The definitions of $x$ and $v$ lead in turn to

$$
\begin{aligned}
& \widetilde{E}_{1}^{\omega}=v \widetilde{E}_{1}^{\nu}+\sqrt{x-v^{2}} \widetilde{E}_{2}^{v}, \\
& \widetilde{E}_{2}^{\omega}=v \widetilde{E}_{2}^{\nu}-\sqrt{x-v^{2}} \widetilde{E}_{1}^{v} .
\end{aligned}
$$

So, given $v, w \in \mathbb{R}, x>v^{2}$, and $\phi$, one can always recover $\widetilde{E}_{L}^{\alpha}$. In addition, Eqs. (2.13) and (2.14) can be seen to imply the identities

$$
\begin{gathered}
K_{\nu}{ }^{\nu}=K-K_{\omega}{ }^{\omega}, \\
K_{\nu}{ }^{\omega}=\left(K-2 K_{\omega}{ }^{\omega}\right) v+K_{\omega}{ }^{\nu} x+J \sqrt{x-v^{2}},
\end{gathered}
$$

that enable us to find the missing components of $K_{\alpha}{ }^{\beta}$ from our new variables. Once $K_{\alpha}{ }^{\beta}$ and $\widetilde{E}_{L}^{\alpha}$ are known, we can finally obtain $A_{\alpha}^{L}$ through

$$
A_{\alpha}^{L}=K_{\alpha}^{\beta}{\underset{\sim}{\beta}}_{\beta}^{L},
$$

$\underset{\sim}{E_{\alpha}^{L}}$ being the inverse of $\widetilde{E}_{L}^{\alpha}$, which can always be computed because $q^{\alpha \beta}$ is positive definite.

As far as we restrict our attention to the sector of Lorentzian nondegenerate metrics, the variables introduced above, together with $A$ and $E$, can then be regarded as a set of elementary variables for our model. Moreover, it is easy to check from Eqs. (2.15) and (2.16) that they form a closed Poisson algebra. The only nonvanishing brackets are

$$
\begin{gathered}
\left\{A(\theta), E\left(\theta^{\prime}\right)\right\}=\left\{J(\theta), \phi\left(\theta^{\prime}\right)\right\}=\left\{K(\theta), w\left(\theta^{\prime}\right)\right\} \\
=i \delta\left(\theta-\theta^{\prime}\right), \\
\left\{K_{\omega}{ }^{\omega}(\theta), K_{\omega}{ }^{\nu}\left(\theta^{\prime}\right)\right\}=-i K_{\omega}{ }^{\nu}(\theta) \delta\left(\theta-\theta^{\prime}\right), \\
\left\{K_{\omega}{ }^{\omega}(\theta), x\left(\theta^{\prime}\right)\right\}=2 i x(\theta) \delta\left(\theta-\theta^{\prime}\right), \\
\left\{K_{\omega}{ }^{\omega}(\theta), v\left(\theta^{\prime}\right)\right\}=i v(\theta) \delta\left(\theta-\theta^{\prime}\right), \\
\left\{K_{\omega}{ }^{\nu}(\theta), x\left(\theta^{\prime}\right)\right\}=2 i v(\theta) \delta\left(\theta-\theta^{\prime}\right), \\
\left\{K_{\omega}{ }^{\nu}(\theta), v\left(\theta^{\prime}\right)\right\}=i \delta\left(\theta-\theta^{\prime}\right) .
\end{gathered}
$$

\section{GAUGE FIXING}

We will now eliminate nonphysical degrees of freedom from our set of phase space variables by introducing suitable gauge-fixing conditions. These conditions, together with the constraints (2.10)-(2.12), will provide us with a set of second-class constraints that will allow one to reduce the model. The gauge-fixing conditions that we are going to impose are

$$
\begin{gathered}
\chi_{H} \equiv E-e^{t}=0, \\
\chi_{G} \equiv \phi=0, \\
\chi_{C} \equiv K-\frac{K_{0}}{\sqrt{2 \pi}}=0,
\end{gathered}
$$

where

$$
K_{0}=\oint \frac{K}{\sqrt{2 \pi}} .
$$

Here, the symbol $\oint$ denotes integration over $\theta \in S^{1}$.

In Eq. (3.1), the time coordinate $t$ is assumed to be real. This condition will be seen to fix the gauge freedom associated with the scalar constraint (2.12). Our gauge fixing is in fact equivalent to Gowdy's choice of time [12] (and, there- 
fore, to that made in Ref. [16]), which can be expressed as $E=\tau, \tau$ being a strictly positive time coordinate. Nonetheless, we notice that, while in Gowdy's time all classical solutions present a cosmological singularity at $\tau=0$ [12], this singularity is driven to minus infinity with our choice of gauge. In this way, we allow a domain of definition for $t$ that is the whole real axis.

We will also prove that the requirement (3.2) fixes completely the Gauss gauge of our model. From Eq. (2.18), this requirement implies $\widetilde{E}_{1}^{\nu}=0$. Finally, we will show that condition (3.3) (which was employed in Ref. [16]) removes almost entirely the diffeomorphism gauge freedom. Some comments are in order concerning the appearance of $K_{0}$ in this gauge-fixing condition. The quantity $K_{0}$ is known to be a classical Dirac observable of the system [20], in the sense that its Poisson brackets with all the first-class constraints (2.10)-(2.12) vanish weakly. As a consequence, $K_{0}$ is a constant of motion whose value depends only on the particular solution that is being considered. This value is invariant under any gauge transformation. On the other hand, since $K$ is a periodic function of $\theta$, it must admit a Fourier series of the form $^{1}$

$$
K=\sum_{n=-\infty}^{\infty} K_{n}(t) \frac{e^{i n \theta}}{\sqrt{2 \pi}}, \quad K_{n}(t)=\oint K \frac{e^{-i n \theta}}{\sqrt{2 \pi}} .
$$

Condition (3.3) amounts thus to absorb all the Fourier coefficients $K_{n}$ with $n \neq 0$ by means of a diffeomorphism.

Let us now see that our gauge-fixing conditions are well posed. A straightforward calculation shows that

$$
\begin{gathered}
\left\{\chi_{H}, \oint_{\sim} \underset{\sim}{n} H\right\}=-2 \underset{\sim}{\operatorname{ma}} E K, \\
\left\{\chi_{G}, \oint \lambda G\right\}=-i \lambda, \\
\left\{\chi_{C}, \oint n C\right\}=i \partial_{\theta}(n K),
\end{gathered}
$$

where $\lambda$ and $n$ are functions on $S^{1}$ and $\underset{\sim}{n}$ is a density of weight -1 . If $\underset{\sim}{n}, \lambda$, and $\partial_{\theta} n$ are different from zero, conditions (3.1) and (3.3) guarantee that these Poisson brackets never vanish for $K_{0} \neq 0$. Therefore, provided that $K_{0}$ does not vanish, our gauge-fixing conditions are second class with the constraints and, hence, acceptable.

The problems found at $K_{0}=0$ can be obviated in the following sense. Using Eqs. (2.2), (2.3), (2.8), (2.9), and (2.13), it is possible to show that the variable $K$ can be equivalently expressed in our model as

$$
K=-i h k_{\alpha \beta} h^{\alpha \beta} .
$$

Here, $h$ is again the square root of the determinant of the three-metric, and $k_{\alpha \beta}$ and $h^{\alpha \beta}$ denote, respectively, the

\footnotetext{
${ }^{1} \mathrm{We}$ assume that all the classical elementary variables, and in particular $K$, are smooth functions of $\theta$. In fact, it suffices that $K \in C^{1}\left(S^{1}\right)$ for its Fourier series to converge to $K$ at all points $\theta \in S^{1}$.
}

$(\alpha \beta)$ components of the extrinsic curvature and the inverse three metric (with $\alpha, \beta=\omega$ or $\nu$ ). Then, $K$ must be purely imaginary if the three-metric is positive definite. Suppose now that $K_{0}=0$. Since $K$ is imaginary and periodic, it follows that it must vanish at least at one point $\theta_{0} \in S^{1}$ on each section of constant time. But one can then easily check that all Poisson brackets of $\chi_{H}$ with the first-class constraints vanish at $\theta_{0}$, modulo such constraints and our gauge-fixing condition. So, our gauge fixing is not admissible if $K_{0}=0$. The same conclusion is reached if one adopts Gowdy's choice of time, $E=\tau$. As a consequence, the classical solutions with $K_{0}=0$, that are not compatible with our gauge fixing, turn out not to be included in the family of cosmologies with the topology of a three-torus studied by Gowdy [12]. Since we are only interested in analyzing this family of solutions, we can disregard the case $K_{0}=0$. Furthermore, we will see in Sec. IV that the geometry of these solutions can be considered invariant under a change of sign in $K_{0}$. Making use of this symmetry, we can set $i K_{0} \in \mathbb{R}^{+}$without loss of generality. In this way, the point $K_{0}=0$ will be driven to the boundary of our reduced phase space. Under quantization, the possible inclusion of that point will be physically irrelevant inasmuch as it will correspond to a set of measure zero in the phase space of the system.

From now on, we will thus take $K_{0} \neq 0$. Equations (3.6)(3.8) ensure then that our conditions (3.1)-(3.3) are suitable to fix the scalar, Gauss, and diffeomorphism gauge degrees of freedom. On the other hand, employing our gauge-fixing conditions, we can solve the scalar and Gauss constraints to obtain the expressions for $A$ and $J$ as functions of the variables $K_{\omega}{ }^{\omega}, K_{\omega}{ }^{\nu}, x, v$, and $K_{0}$ :

$$
\begin{gathered}
A=\frac{\sqrt{2 \pi}}{2 K_{0} \sqrt{x-v^{2}}}\left(K_{\omega}{ }^{\nu} \partial_{\theta} x-2 K_{\omega}{ }^{\omega} \partial_{\theta} v\right)+e^{-t}\left(K_{\omega}{ }^{\nu} v-K_{\omega}{ }^{\omega}\right) \\
+\frac{\sqrt{2 \pi} e^{-t}}{K_{0}}\left[\left(K_{\omega}{ }^{\omega}-K_{\omega}{ }^{\nu} v\right)^{2}+\left(K_{\omega}{ }^{\nu}\right)^{2}\left(x-v^{2}\right)\right], \\
J=0 .
\end{gathered}
$$

This and Eqs. (3.1) and (3.2) remove the two canonically conjugate pairs $(A, E)$ and $(J, \phi)$ as dynamical degrees of freedom.

In addition, the diffeomorphism constraint (2.11) can now be rewritten

$$
\Pi^{\prime}-\frac{K_{0}}{\sqrt{2 \pi}} \partial_{\theta} w=0
$$

where

$$
\Pi^{\prime}=\frac{1}{2\left(x-v^{2}\right)}\left[K_{\omega}{ }^{\nu}\left(v \partial_{\theta} x-2 x \partial_{\theta} v\right)-K_{\omega}{ }^{\omega} \partial_{\theta}\left(x-v^{2}\right)\right] .
$$

Since our fields have a periodic dependence on the angular coordinate $\theta, \Pi$ and $w$ can be expanded as Fourier series similar to that displayed for $K$ in Eq. (3.5). Formula (3.12) fixes then all the Fourier coefficients $w_{n}$ with $n \neq 0$ in terms of $K_{0}$ and the Fourier coefficients of $\Pi^{\prime}$, 


$$
w_{n}=\frac{\sqrt{2 \pi} \Pi_{n}^{\prime}}{i n K_{0}}, \quad n \neq 0 .
$$

The coefficient $w_{0}$ is, however, left undetermined. In addition, integration over $S^{1}$ of Eq. (3.12) leads to the global constraint

$$
\Pi_{0}^{\prime}=\oint \frac{\Pi^{\prime}}{\sqrt{2 \pi}}=0 .
$$

We recall at this point that our gauge-fixing condition (3.3) amounts to set all the Fourier coefficients $K_{n}$ of $K$, except $K_{0}$, equal to zero. On the other hand, Eqs. (2.27)(2.32) imply that $K_{n}$ and $w_{n}$ commute under Poisson brackets with the rest of our phase space variables, whereas

$$
\left\{K_{n}, w_{m}\right\}=i \delta_{-n}^{m}
$$

We conclude in this way that our gauge-fixing condition, together with the diffeomorphism constraint, allow us to eliminate the canonically conjugate pairs $\left(K_{n}, w_{-n}\right)$ with $n$ $\neq 0$ as physical degrees of freedom, while the homogeneous components of $K$ and $w$ (i.e., the Fourier coefficients $K_{0}$ and $w_{0}$ ) remain as dynamical variables. In this reduction process, the diffeomorphism gauge freedom is not totally removed, because we are still left with the homogeneous part of the diffeomorphism constraint, $\Pi_{0}^{\prime}=0$.

In order to prove that our gauge-fixing procedure is consistent, we still have to show that the conditions (3.1)-(3.3) are compatible with the dynamical evolution of the model. This evolution is generated by the total Hamiltonian constraint [2]

$$
H^{T}=\oint\left[-\frac{N}{2} H-i N^{\theta}(C-A G)-i \Lambda G\right],
$$

where $H, C$, and $G$ are the first-class constraints (2.10)(2.12), $\underset{\sim}{N}$ is the densitized lapse function, $N^{\theta}$ is the only nonvanishing component of the shift vector, ${ }^{2}$ and $\Lambda$ is a Lagrange multiplier. In addition, $N$ and $N^{\theta}$ are real if the metric is Lorentzian, and $N$ must be different from zero. What we have to check then is that there exists a choice of densitized lapse, shift, and $\Lambda$ such that the total time derivative of each of our gauge conditions vanishes. This total time derivative (that will be denoted by a dot) is given by the sum of the Poisson brackets with $H^{T},\left\{., H^{T}\right\}$, and the partial derivative with respect to the explicit dependence on the time coordinate $\partial_{t}$. After a careful calculation, we get that, modulo constraints and gauge-fixing conditions,

$$
\begin{gathered}
\dot{\chi}_{H}=e^{t}\left(i \underset{\sim}{N} \frac{K_{0}}{\sqrt{2 \pi}}-1\right), \\
\dot{\chi}_{G}=-\Lambda-i \underset{\sim}{N}\left(\sqrt{x-v^{2}} K_{\omega}{ }^{\nu}-\frac{\sqrt{2 \pi} e^{t}}{K_{0}} \Pi^{\prime}\right)+i e^{t} \partial_{\theta} N,
\end{gathered}
$$

\footnotetext{
${ }^{2}$ The $\omega$ and $\nu$ components of the shift vector can be made equal to zero after the symmetry reduction (2.8) and (2.9).
}

$$
\dot{\chi}_{C}=\frac{K_{0}}{\sqrt{2 \pi}} \partial_{\theta} N^{\theta}
$$

The requirement that $\dot{\chi}_{H}$ vanishes implies

$$
\underset{\sim}{N}=\frac{\sqrt{2 \pi}}{i K_{0}} .
$$

This and $\dot{\chi}_{G}=0$ determine a unique $\Lambda$ through Eq. (3.19). Finally, by demanding that $\dot{\chi}_{C}=0$, we conclude that $N^{\theta}$ can be any function of $t, N^{\theta}=N^{\theta}(t)$. We thus see again that the diffeomorphism gauge freedom has not been entirely removed, since the shift function $N^{\theta}$ is not completely fixed. Any diffeomorphism with infinitesimal parameter $N^{\theta}(t)$ is still allowed. Note that such diffeomorphisms are precisely those generated by the only remaining constraint $\Pi_{0}^{\prime}=0$.

For Lorentzian metrics, the shift $N^{\theta}(t)$ must be real. On the other hand, we have commented above that $K$, and hence $K_{0}$ [from Eq. (3.4)], is purely imaginary in the Lorentzian case. Therefore, the densitized lapse (3.21) is actually real. Moreover, it does not vanish for any finite value of $K_{0}$. This concludes the proof of consistency of our gauge-fixing conditions with the Lorentzian evolution.

The final result of our gauge-fixing procedure is the elimination of the nondynamical fields $(A, E, J, \phi)$ and Fourier coefficients $\left(K_{n}, w_{n}\right)$ with $n \neq 0$. The phase space of the reduced model can be described by the four (periodic) fields $K_{\omega}{ }^{\omega}, K_{\omega}{ }^{\nu}, x$, and $v$, and the two homogeneous variables $K_{0}$ and $w_{0}$. Since all these variables commuted with the nonphysical degrees of freedom that have been suppressed, the reduction of the system does not alter their Poisson brackets (i.e., their Poisson and Dirac brackets coincide). These brackets are given by Eqs. (2.28)-(2.32) and

$$
\left\{K_{0}, w_{0}\right\}=i,
$$

which follows from Eq. (3.16). Finally, the reduced model is still subject to one homogeneous constraint, namely, $\Pi_{0}^{\prime}=0$.

\section{THE REDUCED CLASSICAL MODEL}

In this section, we will analyze the dynamical evolution of our reduced model, discuss the reality conditions on the phase space variables, and obtain the expression of the classical metric.

\section{A. Classical evolution}

We have seen that the shift function $N^{\theta}$ can be any function of time $N^{\theta}(t)$. For practical purposes, nonetheless, this shift function can always be absorbed by replacing the angular coordinate $\theta$ with

$$
\theta^{\prime}=\theta+\int_{t_{0}}^{t} d t^{\prime} N^{\theta}\left(t^{\prime}\right)
$$

where $t_{0}$ is any given time. From the periodicity of $\theta$, it follows that $\theta^{\prime}$ is defined as well on $S^{1}$. Notice also that, for fixed $t, \partial_{\theta^{\prime}}=\partial_{\theta}$. 
Since the vector fields $\left(\partial_{\theta^{\prime}}\right)^{\mu}$ and $\left(\partial_{t}\right)^{\mu}(\mu=t, a)$ are orthogonal, the dynamical evolution (with $\theta^{\prime}$ kept constant) is generated in our model by the reduced Hamiltonian density $H_{R}$, which is provided by the negative of the momentum canonically conjugate to the variable chosen as time. Recalling condition (3.1), which implies that $t=\ln E$, and taking into account the Lorentzian Poisson brackets (2.27)-(2.32), we conclude that the momentum canonically conjugate to our time variable is given by $i A E$. In this way, we arrive at $H_{R}=-i A e^{t}$, with $A$ given by Eq. (3.10). Hence, for constant $\theta^{\prime}$, the time derivative of any of our reduced phase space variables $f$ is

$$
\dot{f}=\partial_{t} f+\left\{f, \oint H_{R}\right\}
$$

In the following, we will assume that the shift $N^{\theta}(t)$ has already been absorbed in $\theta^{\prime}$ and suppress the prime from this angular coordinate.

\section{B. Real phase space variables}

Our variables $\left(x, v, K_{\omega}{ }^{\omega}, K_{\omega}{ }^{\nu}, K_{0}, w_{0}\right)$ present the problem of possessing domains of definition that are rather complicated in the sector of nondegenerate Lorentzian metrics. On the one hand, we know that $x$ must be greater than $v^{2}$, $v$ being real. On the other hand, particularizing to our gaugefixed model the definition of $K_{\alpha}{ }^{\beta}$ in Eq. (2.13) and expressions (2.2) and (2.3) for the Ashtekar connection, it is possible to show that

$$
\begin{gathered}
K_{\omega}{ }^{\omega}=\frac{e^{t}}{4\left(x-v^{2}\right)^{3 / 2}}\left(2 x \partial_{\theta} v-v \partial_{\theta} x\right)-i e^{t / 2} e^{w}\left(x-v^{2}\right)^{1 / 4} k_{\omega}^{\omega}, \\
K_{\omega}{ }^{\nu}=-\frac{e^{t}}{4\left(x-v^{2}\right)^{3 / 2}} \partial_{\theta}\left(x-v^{2}\right)-i e^{t / 2} e^{\omega}\left(x-v^{2}\right)^{1 / 4} k_{\omega}^{\nu} .
\end{gathered}
$$

In these formulas, $k_{a}^{b}$ is the extrinsic curvature, and $w=\Sigma_{n} w_{n} e^{i n \theta} / \sqrt{2 \pi}$, with $w_{n}$ determined by Eq. (3.14) for all $n \neq 0$. So, $K_{\omega}{ }^{\omega}$ and $K_{\omega}{ }^{\nu}$ are not only complex, but their real parts are, in addition, functionally dependent on $x$ and $v$ in an explicitly time-dependent way. We finally recall that, for positive definite three-metrics, the functions $w$ and $K$ have to be real and purely imaginary, respectively. Moreover, we have assumed that $K_{0} \neq 0$. Therefore, we must have $w_{0} \in \mathbb{R}$ and $i K_{0} \in \mathbb{R}^{+} \cup \mathbb{R}^{-}$.

To overcome the above problems, we will now perform a change to a different set of elementary variables whose elements are all real. As a plus, these new variables will form a remarkably simple algebra under Poisson brackets.

Our first step will consist in replacing $x$ with a new metric variable $y$, whose domain of definition is the entire real axis,

$$
y=\ln \left(x-v^{2}\right) .
$$

The condition $x>v^{2}$ ensures that $y$ is real and well defined. Using then Eqs. (2.28)-(2.32), it is not difficult to show that the variables

$$
P_{y}=\frac{i}{2}\left(K_{\omega}{ }^{\omega}-K_{\omega}{ }^{\nu} v\right), \quad P_{v}=i K_{\omega}{ }^{\nu}
$$

are the momenta canonically conjugate to $y$ and $v$,

$$
\left\{y(\theta), P_{y}\left(\theta^{\prime}\right)\right\}=\left\{v(\theta), P_{v}\left(\theta^{\prime}\right)\right\}=\delta\left(\theta-\theta^{\prime}\right) .
$$

The inverse of relations (4.5) and (4.6) is

$$
\begin{gathered}
x=e^{y}+v^{2}, \\
K_{\omega}{ }^{\omega}=-i\left(2 P_{y}+v P_{v}\right), \quad K_{\omega}{ }^{\nu}=-i P_{v} .
\end{gathered}
$$

On the other hand, it is obvious that

$$
k_{0}=i K_{0}
$$

is real and, given Eq. (3.22), canonically conjugate to $w_{0}$,

$$
\left\{w_{0}, k_{0}\right\}=1 .
$$

In addition, $w_{0}$ and $k_{0}$ commute under Poisson brackets with $y, v, P_{y}$, and $P_{v}$. We have hence attained a canonical set of elementary variables for our reduced model.

However, the variables $P_{y}$ and $P_{v}$ are still complex. Actually, it follows from Eqs. (4.3), (4.4), and (4.6) that the real parts of $P_{y}$ and $P_{v}$ run over the whole real axis, whereas their imaginary parts, $\mathcal{I}\left(P_{y}\right)$ and $\mathcal{I}\left(P_{v}\right)$, are restricted to be

$\mathcal{I}\left(P_{y}\right)=\frac{e^{t}}{4} e^{-y / 2} \partial_{\theta} v, \quad \mathcal{I}\left(P_{v}\right)=-\frac{e^{t}}{4} e^{-y / 2} \partial_{\theta} y$.

Nevertheless, it is now easy to arrive at a set of real elementary variables. This can be achieved by means of the canonical tranformation generated by the functional

$$
\mathcal{F}=w_{0} k_{0}^{\prime}+\oint\left[(y-2 t) p_{u}+v p_{v}\right]+i F,
$$

where

$$
F=-\frac{e^{t}}{2} \oint e^{-y / 2} \partial_{\theta} v
$$

and $p_{u}(\theta), p_{v}(\theta)$, and $k_{0}^{\prime}$ are the new momenta. It is straightforward to check that $k_{0}=k_{0}^{\prime}$ and that our configuration variables $v(\theta)$ and $w_{0}$ are not affected by the transformation. The field $y(\theta)$ is, however, replaced with a new configuration variable $u(\theta)$, whose domain of definition is given again by the real axis

$$
u=y-2 t .
$$

One can see that this change of variable partly simplifies the explicit time dependence of the metric. More importantly, since $F$ satisfies

$$
\frac{\delta F}{\delta y}=\mathcal{I}\left(P_{y}\right), \quad \frac{\delta F}{\delta v}=\mathcal{I}\left(P_{v}\right),
$$

the new momenta $p_{u}$ and $p_{v}$ turn out to coincide with the real parts of $P_{y}$ and $P_{v}$, respectively,

$$
P_{y}=p_{u}+i \mathcal{I}\left(P_{y}\right), \quad P_{v}=p_{v}+i \mathcal{I}\left(P_{v}\right) .
$$


Therefore, $\left(u, p_{u}, v, p_{v}\right)$ (which are fields on $S^{1}$ ) and $\left(w_{0}, k_{0}\right)$ provide a canonical set of real elementary variables for our reduced model. All of these variables run over the whole real axis, except $k_{0}$, which has to be nonvanishing. In addition, the model possesses one homogeneous constraint, that is given by Eq. (3.15). After some calculus, we can express it as

$$
\begin{gathered}
\Pi_{0}=-i \Pi_{0}^{\prime}=\oint \frac{\Pi}{\sqrt{2 \pi}}=0, \\
\Pi=-i \Pi^{\prime}=\partial_{\theta} u p_{u}+\partial_{\theta} v p_{v} .
\end{gathered}
$$

This constraint reproduces the periodicity condition found by Gowdy [12]. In fact, it was obtained from Eq. (3.12) by assuming that the function $w$ is periodic. As remarked by Gowdy, it can also be interpreted as the condition that the "total field momentum be zero" [12].

Let us finally notice that, since the generating functional $\mathcal{F}$ of the above canonical transformation is explicitly time dependent, the dynamical evolution of the variables $\left(u, p_{u}, v, p_{v}, w_{0}, k_{0}\right)$ is not generated by the Hamiltonian $H_{R}$ anymore. The new reduced Hamiltonian $H_{r}$ can be obtained from the standard formula

$$
\oint H_{r}=\oint H_{R}+\partial_{t} \mathcal{F}
$$

Using that $\partial_{t} \mathcal{F}=-2 \oint p_{u}+i F$ and $H_{R}=-i A e^{t}$, with $A$ given by Eq. (3.10), we get

$$
H_{r}=-\frac{\sqrt{2 \pi}}{k_{0}}\left[4 p_{u}^{2}+e^{2 t} e^{u} p_{v}^{2}+\frac{e^{2 t}}{16}\left(\partial_{\theta} u\right)^{2}+\frac{e^{-u}}{4}\left(\partial_{\theta} v\right)^{2}\right] .
$$

It is straightforward to see that this Hamiltonian is bounded from above (below) for positive (negative) values of $k_{0}$.

\section{The metric}

We will now obtain the expression of the classical metric that results from our gauge-fixing conditions for the Gowdy model. Using Eqs. (2.2), (2.8), (2.17)-(2.19), and the definition of $u$, we get that the only nonvanishing components of the three-metric $h_{a b}$ are

$$
\begin{gathered}
h_{\theta \theta}=e^{2 w} e^{u / 2}, \quad h_{\alpha \beta}=e^{-u / 2} g_{\alpha \beta}, \\
g_{\omega \omega}=1, \quad g_{\omega \nu}=-v, \quad g_{\nu \nu}=e^{2 t} e^{u}+v^{2} .
\end{gathered}
$$

The lapse function, $N=N\left(\operatorname{det} h_{a b}\right)^{1 / 2}$, can then be found from Eq. (3.21),

$$
N=\frac{\sqrt{2 \pi}}{k_{0}} e^{t} e^{w} e^{u / 4}
$$

Introducing now the change of time coordinate

$$
T=\frac{\sqrt{2 \pi}}{\left|k_{0}\right|} e^{t}, \quad T \in \mathbb{R}^{+},
$$

and remembering that the shift function $N^{\theta}$ has been absorbed in the angular coordinate $\theta$, we arrive at a line element of the form

$$
\begin{aligned}
d s^{2}= & e^{2 w} e^{u / 2}\left(-d T^{2}+d \theta^{2}\right)+e^{-u / 2}\left(g_{\omega \omega} d \omega^{2}\right. \\
& \left.+2 g_{\omega \nu} d \omega d \nu+g_{\nu \nu} d \nu^{2}\right)
\end{aligned}
$$

where, in terms of the positive time coordinate $T, g_{\nu \nu}$ reads

$$
g_{\nu \nu}=\frac{k_{0}^{2}}{2 \pi} T^{2} e^{u}+v^{2} .
$$

On the other hand, Eq. (3.14) determines $w$ to be

$$
w=\frac{w_{0}}{\sqrt{2 \pi}}-\sum_{n=-\infty, \neq 0}^{\infty} \frac{\Pi_{n}}{\operatorname{ink}_{0}} e^{i n \theta},
$$

with $\Pi_{n}$ the Fourier coefficients for $\Pi$, and $\Pi_{0}=0$ because of constraint (4.18). We have thus succeeded in writing the metric of our model in terms of the dynamical variables $u$, $p_{u}, v, p_{v}, w_{0}$, and $k_{0}$.

It is easy to check that the classical geometries described by Eqs. (4.26)-(4.28) are in fact invariant under a flip of sign in the momenta $p_{u}, p_{v}$, and $k_{0} .{ }^{3} \mathrm{We}$ can take advantage of this symmetry to fix, e.g., the sign of $k_{0}$, without eliminating from our considerations any of the geometries that are allowed for our model. We will hence restrict $k_{0}$ to be positive from now on, $k_{0} \in \mathbb{R}^{+}$.

Given this restriction, it is convenient to replace $\left(w_{0}, k_{0}\right)$ with a new pair of canonical variables $\left(b_{0}, c_{0}\right)$, whose respective domains of definition are the whole real axis,

$$
b_{0}=k_{0} w_{0}, \quad c_{0}=\ln k_{0} .
$$

Note that

$$
\left\{b_{0}, c_{0}\right\}=1,
$$

and that the point $k_{0}=0$, excluded from our phase space, has now been driven to the boundary of the domain of $c_{0}$ (namely, to $c_{0}=-\infty$ ). Notice also that, after performing the change (4.29), the Hamiltonian (4.21) turns out to be analytic in all the elementary fields and variables of our reduced model.

To close this section, we will derive a formula equivalent to Eq. (4.28) that may be more useful in practice to compute the metric function $w$. A straightforward calculation leads to

$$
\begin{gathered}
\dot{\Pi}=\left\{\Pi, \oint H_{r}\right\}=\partial_{\theta} H_{r}, \\
\dot{w}_{0}=\left\{w_{0}, \oint H_{r}\right\}=-\frac{1}{k_{0}} \oint H_{r} .
\end{gathered}
$$

It is worth remarking that the first of these equations implies that the constraint (4.18) is preserved by the dynamical evolution of our model. From Eqs. (4.31) and (4.32), we also get

\footnotetext{
${ }^{3}$ This change of sign in the momenta, while keeping unaltered the configuration variables, can be interpreted as a time reversal.
} 
the following relations among $w_{0}$ and the Fourier coefficients of $\Pi$ and $H_{r}\left(\Pi_{n}\right.$ and $H_{r}^{n}$, respectively):

$$
\begin{aligned}
\frac{\Pi_{n}}{i n} & =\int_{0}^{t} d t^{\prime} H_{r}^{n}\left(t^{\prime}\right)+d_{n}, \quad n \neq 0, \\
w_{0} & =-\frac{\sqrt{2 \pi}}{k_{0}} \int_{0}^{t} d t^{\prime} H_{r}^{0}\left(t^{\prime}\right)+d_{0},
\end{aligned}
$$

the $d_{n}$ 's being constants for all integers $n$. Using these relations, it is not difficult to check that Eq. (4.28) can be rewritten as

$$
\begin{aligned}
w(t, \theta)= & -\frac{\sqrt{2 \pi}}{k_{0}}\left[\int_{0}^{t} d t^{\prime} H_{r}\left(t^{\prime}, \theta=0\right)\right. \\
& \left.+\int_{0}^{\theta} d \theta^{\prime} \Pi\left(t, \theta^{\prime}\right)\right]+D,
\end{aligned}
$$

where $D$ is an undetermined real constant.

\section{QUANTIZATION}

We have seen that the four fields $\left(u, p_{u}, v, p_{v}\right)$ and the two homogeneous degrees of freedom $\left(b_{0}, c_{0}\right)$ form a set of real elementary variables for our reduced model. They are only restricted in that they are subject to the constraint (4.18). Since all our elementary fields are defined on $S^{1}$, we can expand them as Fourier series. The corresponding Fourier coefficients will be called $\left(u_{n}, p_{u}^{n}, v_{n}, p_{v}^{n}\right)$. These coefficients, together with $\left(b_{0}, c_{0}\right)$, provide then an infinite set of homogeneous, elementary variables for our system. From the fact that $\left(p_{u}, p_{v}\right)$ are the momenta canonically conjugate to $(u, v)$, we arrive at the following Poisson bracket structure:

$$
\left\{u_{n}, p_{u}^{m}\right\}=\left\{v_{n}, p_{v}^{m}\right\}=\delta_{-n}^{m}, \quad\left\{b_{0}, c_{0}\right\}=1,
$$

the rest of brackets being equal to zero. On the other hand, the reality conditions on our fields $\left(u, p_{u}, v, p_{v}\right)$ and variables $\left(b_{0}, c_{0}\right)$ imply that

$$
\overline{b_{0}}=b_{0}, \quad \overline{c_{0}}=c_{0}, \quad \overline{g_{n}}=g_{-n}, \quad \overline{p_{g}^{n}}=p_{g}^{-n},
$$

where $g=u$ or $v$ and the bar denotes complex conjugation.

In order to quantize the system, we will follow the canonical quantization program put forward by Ashtekar [2]. We will first represent our elementary variables by linear operators acting on an auxiliary vector space. An inner product will be selected on this space by imposing the reality conditions (5.2) as adjointness relations between our operators $[2,17]$. We will then represent the constraint (4.18) as an operator $\hat{\Pi}_{0}$, and impose it on our quantum theory. From the kernel of $\hat{\Pi}_{0}$ and the inner product introduced on the auxiliary representation space, we will construct the Hilbert space of physical states $\mathcal{H}_{p}$. Finally, we will identify the quantum observables of our reduced model. By quantum observable, we mean any operator that has a well-defined action on $\mathcal{H}_{p}$ and, therefore, commutes with the only constraint of the model, $\hat{\Pi}_{0}$. In the rest of this section, we will implement this quantization program, except for the discussion of the quan- tum observables, that will be postponed to Sec. VI. At some stages of our analysis, we will proceed only in a formal way; thus, our main concern is to show how the quantization process can be carried out, rather than obtain explicitly a rigorous and complete quantum theory for our model.

We begin by choosing as our auxiliary representation space the vector space of analytic functionals $\Psi$ of the set of variables $\Omega \equiv\left(c_{0}, u_{n}, v_{n}\right)[n=0, \pm 1, \ldots]$. On this space, we can represent our elementary variables by the operators

$$
\begin{gathered}
\hat{c}_{0} \Psi=c_{0} \Psi, \quad \hat{b}_{0} \Psi=i \frac{\partial \Psi}{\partial c_{0}}, \\
\hat{g}_{n} \Psi=g_{n} \Psi, \quad \hat{p}_{g}^{-n} \Psi=-i \frac{\partial \Psi}{\partial g_{n}},
\end{gathered}
$$

where $g$ stands again for $u$ or $v$ (and we have set $\hbar=1$ ). Notice that the commutators of these operators reproduce the classical Poisson algebra (5.1) with the due factor of $i$ :

$$
\left[\hat{b}_{0}, \hat{c}_{0}\right]=i, \quad\left[\hat{g}_{n}, \hat{p}_{g}^{-n}\right]=i
$$

The operators of our quantum theory are then given by (possibly infinite) sums of products of the elementary operators (5.3) and (5.4) and $c$-number operators [2].

Using the reality conditions (5.2), we can select an inner product on our auxiliary representation space in the following way. We first adopt the ansatz

$$
\langle\Phi, \Psi\rangle=\int d \Omega \wedge d \bar{\Omega} \rho(\Omega, \bar{\Omega}) \overline{\Phi(\Omega)} \Psi(\Omega),
$$

with

$$
\begin{aligned}
d \Omega \wedge d \bar{\Omega} \equiv & \frac{i}{2} d c_{0} \wedge d \overline{c_{0}} \prod_{n}\left(\frac{i}{2} d u_{n} \wedge d \overline{u_{n}}\right) \\
& \times \prod_{m}\left(\frac{i}{2} d v_{m} \wedge d \overline{v_{m}}\right)
\end{aligned}
$$

and $\rho$ a certain positive integration measure. The requirement that the reality conditions $(5.2)$ be realized as adjointness relations determines then the measure $\rho$ up to an overall positive constant,

$$
\rho=\delta\left(c_{0}-\overline{c_{0}}\right) \prod_{n}\left[\delta\left(u_{-n}-\overline{u_{n}}\right) \delta\left(v_{-n}-\overline{v_{n}}\right)\right]
$$

In this formula, we have employed the notation

$$
\delta(z) \delta(\vec{z}) \equiv \delta(x) \delta(y), \quad \delta(z-\vec{x}) \equiv \delta(y)
$$

for $z=x+i y$ any complex variable, and $x, y \in \mathbb{R}$.

We still have to impose the homogeneous constraint (4.18) quantum mechanically. In order to do it, let us represent the phase space variable $\Pi_{0}$ by the operator

$$
\hat{\Pi}_{0}=\sum_{s=1}^{\infty} s\left(\hat{X}_{s}^{u}+\hat{X}_{s}^{v}\right)
$$

where (for $g=u$ or $v$ ) 


$$
\hat{X}_{s}^{g}=i\left(\hat{g}_{s} \hat{p}_{g}^{-s}-\hat{g}_{-s} \hat{p}_{g}^{s}\right) .
$$

We note that, with the factor ordering chosen in Eq. (5.11), $\hat{\Pi}_{0}$ is, at least formally, self-adjoint on the auxiliary Hilbert space determined by the inner product (5.6)-(5.8).

The physical states of our quantum theory are those annihilated by the constraint operator $\hat{\Pi}_{0}$. In our auxiliary representation space, on the other hand, all quantum states $\Psi$ can be expressed as (possibly infinite) sums of functionals of the form

$$
P_{(k, \sigma)}=c_{0}^{k} \prod_{n}\left(u_{n}^{i_{n}} v_{n}^{j_{n}}\right),
$$

where $\sigma \equiv\left(i_{n}, j_{n}\right)[n=0, \pm 1, \ldots]$, and $k, i_{n}$, and $j_{n}$ are nonnegative integers. From Eqs. (5.10) and (5.11), it is easy to check that the functionals $P_{(k, \sigma)}$ are (generalized) eigenfunctions of $\hat{\Pi}_{0}$,

$$
\hat{\Pi}_{0} P_{(k, \sigma)}=N(\sigma) P_{(k, \sigma)} .
$$

Here, $N(\sigma)$ is given by

$$
N(\sigma)=\sum_{s=1}^{\infty} s\left(i_{s}+j_{s}-i_{-s}-j_{-s}\right)
$$

and is equal to the sum of indices of all factors $\left(u_{n}, v_{n}\right)$ (including multiplicities) appearing in $P_{(k, \sigma)}$. For any quantum state $\Psi=\sum a_{(k, \sigma)} P_{(k, \sigma)}$, with $a_{(k, \sigma)}$ some complex constants, we, therefore, get

$$
\hat{\Pi}_{0} \Psi=\sum_{(k, \sigma)} a_{(k, \sigma)} N(\sigma) P_{(k, \sigma)} .
$$

The uniqueness of the power series of the zero functional implies then that $\Psi$ is annihilated by $\hat{\Pi}_{0}$ if and only if it is a (generally infinite) linear combination of functionals $P_{(k, \sigma)}$ whose total indices $N(\sigma)$ vanish. The analytic functionals that satisfy this condition form a complex vector space, whose Hilbert completion with respect to the inner product (5.6)-(5.8) finally provides us with the Hilbert space of physical states $\mathcal{H}_{p}$.

It is easy to see that this Hilbert space is actually infinite dimensional. For the sake of an example, let us display an infinite set of states in $\mathcal{H}_{p}$, namely,

$$
\begin{aligned}
\Psi= & P_{\left(k, \sigma^{\prime}\right)} \frac{1}{C}\left(\prod_{s \geqslant 0} \frac{1}{2 \pi A_{s} B_{s}}\right) \exp \left\{-\frac{1}{4}\left[\frac{c_{0}^{2}}{C^{2}}\right.\right. \\
& \left.\left.+\sum_{s=0}^{\infty}\left(\frac{u_{s} u_{-s}}{A_{s}^{2}}+\frac{v_{s} v_{-s}}{B_{s}^{2}}\right)\right]\right\},
\end{aligned}
$$

where $C, A_{s}$, and $B_{s}(s=0,1, \ldots)$ are real constants, but otherwise unrestricted, and $P_{\left(k, \sigma^{\prime}\right)}$ is any polynomial of the form (5.12) (i.e., the set $\sigma^{\prime}$ contains only a finite number of nonvanishing elements in this case) such that $N\left(\sigma^{\prime}\right)=0$. All these states are analytic functionals of $\Omega$, belong to the kernel of $\hat{\Pi}_{0}$, and can be checked to be normalizable with respect to the inner product (5.6)-(5.8).

\section{QUANTUM OBSERVABLES AND HAMILTONIAN}

We turn now to the task of finding the quantum observables of our model. These are the operators with a welldefined action on the Hilbert space of physical states $\mathcal{H}_{p}$. As they leave $\mathcal{H}_{p}$ invariant, they must (weakly) commute with the quantum constraint of the model $\hat{\Pi}_{0}$.

In our quantum theory, on the other hand, all operators are supposed to be constructed from the elementary operators (5.3) and (5.4) by taking (suitable limits of) sums and products. In particular, it should be possible to obtain all quantum observables from (infinite) linear combinations of operators of the form

$$
\hat{P}_{(k, \Gamma)}=\hat{c}_{0}^{k_{1}} \hat{b}_{0}^{k_{2}} \prod_{n}\left[\hat{u}_{n}^{i_{n}} \hat{v}_{n}^{j_{n}}\left(\hat{p}_{u}^{-n}\right)^{l_{-n}}\left(\hat{p}_{v}^{-n}\right)^{m_{-n}}\right],
$$

$k \equiv\left(k_{1}, k_{2}\right)$ and $\Gamma \equiv\left(i_{n}, j_{n}, l_{n}, m_{n}\right) \quad[n=0, \pm 1, \ldots]$ being two sets of non-negative integers.

A straightforward calculation shows that

$$
\left[\hat{\Pi}_{0}, \hat{P}_{(k, \Gamma)}\right]=N(\Gamma) \hat{P}_{(k, \Gamma)},
$$

where

$$
N(\Gamma)=\sum_{s=1}^{\infty} s\left(i_{s}+j_{s}+l_{s}+m_{s}-i_{-s}-j_{-s}-l_{-s}-m_{-s}\right)
$$

is [similarly to $N(\sigma)$ in Eq. (5.14) ] the sum of indices of all the elementary operators (counting multiplicities) that form $\hat{P}_{(k, \Gamma)}$. Therefore, $\hat{P}_{(k, \Gamma)}$ commutes with $\hat{\Pi}_{0}$ if and only if its total index $N(\Gamma)$ vanishes. Furthermore, from Eq. (6.2) and our comments above, it is possible to show that the quantum observables of our theory can always be expressed ${ }^{4}$ as linear combinations (including the limit of infinite sums) of operators $\hat{P}_{(k, \Gamma)}$ verifying $N(\Gamma)=0$.

A possible way to attain observables is the following. We first define

$$
\hat{g}(\theta)=\sum_{n=-\infty}^{\infty} \hat{g}_{n} \frac{e^{i n \theta}}{\sqrt{2 \pi}}, \quad \hat{p}_{g}(\theta)=\sum_{n=-\infty}^{\infty} \hat{p}_{g}^{n} \frac{e^{i n \theta}}{\sqrt{2 \pi}} .
$$

Here, $g=u$ or $v$, and $\theta \in S^{1}$ must be regarded as a parameter. Suppose next that $g\left(c_{0}, b_{0}\right)$ is an analytic function of $c_{0}$ and $b_{0}$, and $f[u(\theta), v(\theta)]$ a functional of only $u(\theta)$ and $v(\theta)$ (and perhaps of their derivatives with respect to $\theta$ ) which is analytic in these fields. One can then check that, for all nonnegative integers $l$ and $m$, the operators

$$
g\left(\hat{c}_{0}, \hat{b}_{0}\right) \oint f[\hat{u}, \hat{v}]\left(\hat{p}_{u}\right)^{l}\left(\hat{p}_{v}\right)^{m}
$$

\footnotetext{
${ }^{4} \mathrm{Up}$ to additive terms of the form $\hat{X} \hat{\Pi}_{0}(\hat{X}$ being a generic operator), which vanish modulo the constraint $\hat{\Pi}_{0}$.
} 
can be written as (infinite) sums of operators $\hat{P}_{(k, \Gamma)}$ with $N(\Gamma)=0$, so that they provide, in general, quantum observables for our model.

So far, we have not discussed the dynamics of our quantum system. In order to do it, we must first allow the physical states and quantum observables to depend on a dynamical parameter $t \in \mathbb{R}$. The quantum evolution is then dictated by the Schrödinger equation

$$
i \frac{\partial \Psi}{\partial t}(t)=\hat{H}_{r}^{T}(t) \Psi(t),
$$

where $\hat{H}_{r}^{T}$ is, by assumption, a self-adjoint observable that represents the classical reduced Hamiltonian integrated over $S^{1}$, that is, the generator of the classical evolution $\oint H_{r}$.

The self-adjointness of $\hat{H}_{r}^{T}$ implies that the quantum evolution is unitary, i.e., it preserves the norm of the physical states of the theory. This is equivalent to say that the integration of the Schrödinger equation (6.6) leads then to a unitary evolution operator $\hat{U}(t, 0)$, such that $\Psi(t)$ $=\hat{U}(t, 0) \Psi, \Psi$ being the initial physical state. In terms of the quantum Hamiltonian, the evolution operator adopts the expression [21]

$$
\hat{U}(t, 0)=\mathcal{P}\left[\exp \left(-i \int_{0}^{t} d t^{\prime} \hat{H}_{r}^{T}\left(t^{\prime}\right)\right)\right],
$$

where $\mathcal{P}$ stands for the time ordering

$$
\begin{aligned}
\mathcal{P}\left[\hat{H}_{r}^{T}\left(t_{1}\right) \cdots \hat{H}_{r}^{T}\left(t_{n}\right)\right]= & \sum_{(\eta)} \hat{H}_{r}^{T}\left(t_{\eta(1)}\right) \cdots \hat{H}_{r}^{T}\left(t_{\eta(n)}\right) \Theta\left(t_{\eta(1)}\right. \\
& \left.-t_{\eta(2)}\right) \cdots \Theta\left(t_{\eta(n-1)}-t_{\eta(n)}\right) .
\end{aligned}
$$

Here, $\Theta$ is the Heaviside function and $\eta$ any permutation of the indices $1, \ldots, n$.

From the Schrödinger equation and the self-adjointness of the quantum Hamiltonian, we also arrive at the following evolution for the matrix elements of any quantum observable $\hat{O}(t)$ :

$$
\begin{aligned}
i \frac{d}{d t}\langle\Phi(t), \hat{O}(t) \Psi(t)\rangle= & \left\langle\Phi(t),\left\{\left[\hat{O}(t), \hat{H}_{r}^{T}(t)\right]\right.\right. \\
& \left.\left.+i \partial_{t} \hat{O}(t)\right\} \Psi(t)\right\rangle
\end{aligned}
$$

with $\partial_{t} \hat{O}(t)$ the derivative of $\hat{O}(t)$ with respect to its explicit dependence on the parameter $t$. We will then say that an observable $\hat{O}(t)$ represents a constant of motion if it satisfies

$$
\left[\hat{O}(t), \hat{H}_{r}^{T}(t)\right]+i \partial_{t} \hat{O}(t)=0,
$$

so that all its matrix elements are constant in the quantum evolution. In this sense, it is worth pointing out that, given any quantum observable $\hat{O}$, that is explicitly $t$ independent, one can generally obtain another observable that represents a constant of motion, namely,

$$
\hat{O}^{\prime}(t)=\hat{U}(t, 0) \hat{O} \hat{U}^{-1}(t, 0),
$$

where $\hat{U}^{-1}(t, 0)$ is the inverse of the evolution operator.

We have thus seen that, in order to arrive at a unitary quantum evolution and essentially complete our quantization, we are only left with the problem of finding a selfadjoint observable to represent the (integrated) classical reduced Hamiltonian of the model. A quantum Hamiltonian that, at least formally, satisfies these conditions is

$$
\begin{gathered}
\hat{H}_{r}^{T}=-\hat{X}-e^{2 t} \hat{Y} \\
\hat{X}=\sqrt{2 \pi} e^{-\hat{c}_{0}} \oint\left[4\left(\hat{p}_{u}\right)^{2}+\frac{1}{4} e^{-\hat{u}}\left(\partial_{\theta} \hat{v}\right)^{2}\right] \\
=e^{-\hat{c}_{0}} \sum_{n=-\infty}^{\infty}\left[4 \sqrt{2 \pi} \hat{p}_{u}^{n} \hat{p}_{u}^{-n}-\sum_{m=-\infty}^{\infty} \frac{n m}{4} e_{-n-m}^{-\hat{u}} \hat{v}_{n} \hat{v}_{m}\right], \\
\hat{Y}=\sqrt{2 \pi} e^{-\hat{c}_{0}} \oint\left[\frac{1}{16}\left(\partial_{\theta} \hat{u}\right)^{2}+e^{\hat{u}}\left(\hat{p}_{v}\right)^{2}\right] \\
=e^{-\hat{c}_{0}} \sum_{n=-\infty}^{\infty}\left[\frac{\sqrt{2 \pi}}{16} n^{2} \hat{u}_{n} \hat{u}_{-n}+\sum_{m=-\infty}^{\infty} e_{-n-m}^{\hat{u}} \hat{p}_{v}^{n} \hat{p}_{v}^{m}\right] .
\end{gathered}
$$

In the above formulas,

$$
e_{n}^{ \pm \hat{u}}=\oint e^{ \pm \hat{u}} \frac{e^{-i n \theta}}{\sqrt{2 \pi}}
$$

and $\hat{u}, \hat{p}_{u}$, and $\hat{p}_{v}$ are the operators defined in Eq. (6.4). It is clear that this quantum Hamiltonian commutes with $\hat{\Pi}_{0}$, because it is a linear combination of operators of the form (6.5). That this Hamiltonian is formally self-adjoint follows from the fact that it is given by a sum of products of commuting operators, as well as from the reality conditions $\hat{c}_{0}^{\dagger}=\hat{c}_{0}, \hat{g}_{n}^{\dagger}=\hat{g}_{-n}$, and $\left(\hat{p}_{g}^{n}\right)^{\dagger}=\hat{p}_{g}^{-n}(g=u$ or $v)$. Finally, notice that $\hat{H}_{r}^{T}$ inherits an explicit dependence on the parameter $t$ from the time dependence of the classical Hamiltonian (4.21).

To prove that the Hamiltonian (6.12)-(6.14) is in fact a self-adjoint observable, it would actually suffice to show that it is densely defined on the Hilbert space of physical states $\mathcal{H}_{p}$. From our discussion above, this would guarantee that $\hat{H}_{r}^{T}$ is a symmetric observable. That this Hamiltonian is selfadjoint (or, strictly speaking, that it admits a self-adjoint extension) would then be a consequence of the fact that there exists a conjugation $\mathcal{C}$ on $\mathcal{H}_{p}$ which leaves the domain of $\hat{H}_{r}^{T}$ invariant and commutes with it [22]. We remind that a conjugation $\mathcal{C}: \mathcal{H}_{p} \rightarrow \mathcal{H}_{p}$ is an antilinear, norm-preserving map whose square is the identity. It is not difficult to check that a map on $\mathcal{H}_{p}$ that satisfies the properties of a conjugation and commutes with our quantum Hamiltonian is

$$
\mathcal{C} \Psi(\Omega)=\bar{\Psi}(\mathcal{C}(\Omega))
$$

where $\Psi$ is any physical state and the action of $\mathcal{C}$ on the set of elementary variables $\Omega$ is given by 


$$
\mathcal{C} c_{0}=c_{0}, \quad \mathcal{C} u_{n}=u_{-n}, \quad \mathcal{C} v_{n}=v_{-n},
$$

with $n=0, \pm 1, \ldots$.

It could also happen that, instead on $\mathcal{H}_{p}$, the Hamiltonian (6.12) - (6.14) admitted a self-adjoint extension only on a sufficiently large Hilbert subspace $\mathcal{H}_{p}^{1} \subset \mathcal{H}_{p}$. In that case, one could still try to restrict all considerations to that subspace in a consistent way, and regard $\mathcal{H}_{p}^{1}$ as the true Hilbert space of physical states. Otherwise, one would have to replace the operator (6.12)-(6.14) with a different quantum Hamiltonian that turned out to be physically acceptable in our model. Finally, if no such Hamiltonian could be found (and one insisted in arriving at a unitary quantum evolution), one would have to start the quantization over again, changing any of the choices that are available in the construction of the quantum theory, such as, e.g., the set of elementary operators or their representation.

\section{CONCLUSIONS AND FURTHER COMMENTS}

Starting with the Ashtekar formalism for Lorentzian general relativity in vacuum and restricting our attention to the sector of nondegenerate metrics, we have discussed the structure of the reduced phase space and the quantization of the family of Gowdy universes whose spatial topology is that of a three-torus.

We have first removed nonphysical degrees of freedom by means of a gauge-fixing procedure. The gauge-fixing conditions imposed, together with the first-class constraints of the model, have been shown to form a set of second-class constraints that allow the reduction of the system. In this way, we have been able to eliminate all the constraints of the model except for one homogeneous constraint $\Pi_{0}=0$. This constraint is the analogue of the periodicity condition studied by Gowdy [12], and generates the diffeomorphisms, with spatially constant infinitesimal parameters, of the angular coordinate $\theta$ that does not correspond to a Killing field of the spacetime.

The choice of time that we have adopted is equivalent to that employed by Gowdy $[12,16]$. We have got rid of the Gauss constraints and of the diffeomorphism constraints of the coordinates associated with Killing fields by requiring that some components of the densitized triad vanish [see Eqs. (2.8), (2.18), and (3.2)]. Finally, the $\theta$-coordinate diffeomorphism gauge freedom has been used to set the variable $K$ [given by Eq. (3.9)] equal to its mean value on each surface of constant time [16], i.e., to $K_{0} / \sqrt{2 \pi}$. This quantity is known to be a constant of motion of the model. We have then shown that the classical geometries with $K_{0}=0$ are not included in the family of cosmological solutions considered by Gowdy. In addition, provided that $K_{0}$ is different from zero, our gauge-fixing conditions are consistent and wellposed.

We have found a canonical set of real elementary variables for the phase space of our reduced model. This set is formed by the four fields $u(\theta), p_{u}(\theta), v(\theta)$, and $p_{v}(\theta)$, and by the two homogeneous variables $w_{0}$ and $k_{0}$. The reduced model is still subject to the homogeneous constraint $\Pi_{0}=0$. On the other hand, the exclusion of the solutions with $K_{0}=0$ implies that $k_{0} \in \mathbb{R}^{+} \cup \mathbb{R}^{-}$. Making use of the fact that the classical geometries are invariant under a change of sign in the momenta $p_{u}(\theta), p_{v}(\theta)$, and $k_{0}$, we have nonetheless been able to restrict all considerations to the case $k_{0} \in \mathbb{R}^{+}$without loss of generality. In order to attain a canonical set of elementary variables whose respective domains of definition are the entire real axis, we have then replaced $w_{0}$ and $k_{0}$ with a new canonical pair of variables $\left(b_{0}, c_{0}\right)$.

In addition, we have obtained the explicit expression for the classical metric of the Gowdy spacetimes and determined the reduced Hamiltonian $H_{r}$, that generates the dynamical evolution in our gauge-fixed model. This Hamiltonian presents an explicit dependence on the time coordinate, so that the reduced system is not conservative.

Since the fields $u(\theta), p_{u}(\theta), v(\theta)$, and $p_{v}(\theta)$ are periodic functions of $\theta$, we can expand them as Fourier series. The Fourier coefficients $\left(u_{n}, p_{u}^{-n}\right)$ and $\left(v_{n}, p_{v}^{-n}\right)$ turn out to be canonically conjugate pairs of homogeneous variables. Employing these Fourier coefficients and $\left(b_{0}, c_{0}\right)$ as elementary variables, we have proceeded to quantize our model following the canonical program elaborated by Ashtekar [2]. We have first represented the variables $b_{0}, c_{0}, u_{n}, p_{u}^{n}, v_{n}$, and $p_{v}^{n}(n=0, \pm 1, \ldots)$ as elementary linear operators acting on the vector space of analytic functionals of $c_{0}, u_{n}$, and $v_{n}$. A unique inner product has been selected on this space by demanding that the complex conjugation relations (5.2) (our reality conditions) are realized quantum mechanically as adjointness relations. We have then represented the homogeneous constraint of our reduced model by a linear operator $\hat{\Pi}_{0}$, and determined the quantum states that are annihilated by it. These states, together with the inner product selected by the reality conditions, have provided us with the Hilbert space of physical states $\mathcal{H}_{p}$.

The quantum observables of the reduced model are the operators that have a well-defined action on $\mathcal{H}_{p}$. In our quantum theory, on the other hand, a generic observable should always be given by a suitable (possibly infinite) sum of products of elementary operators. Using this fact, we have been able to obtain the general form of the quantum observables.

We have finally introduced a dynamical evolution in our system by imposing a Schrödinger equation with quantum Hamiltonian $\hat{H}_{r}^{T}$, representing the classical reduced Hamiltonian integrated over $S^{1}$. If one requires that the quantum evolution be unitary, the Hamiltonian $\hat{H}_{r}^{T}$ must be a selfadjoint observable. We have found an operator $\hat{H}_{r}^{T}$ that, at least formally, satisfies these conditions. Also discussed are other still available, alternative possibilities to obtain a Hamiltonian which would really be well defined and selfadjoint.

In analyzing the structure of the phase space of our reduced model, we have restricted the variable $k_{0}$ to be positive by taking advantage of the symmetry of the classical geometries under a change of sign in the momenta $p_{u}(\theta)$, $p_{v}(\theta)$, and $k_{0}$, a transformation that can be regarded as a time reversal. Had we not imposed this restriction, we should have split the phase space into two disconnected parts: one for $k_{0}>0$, and the other for $k_{0}<0$. Replacing definiton (4.29) with

$$
b_{0}=k_{0} w_{0}, \quad c_{0}=\ln \left(-k_{0}\right)
$$


in the sector of negative values of $k_{0}$ and repeating our quantization procedure, we would have then arrived at a quantum theory whose physical Hilbert space would be given by the direct sum of two copies of the Hilbert space of physical states constructed for $k_{0} \in \mathbb{R}^{+}$. Nevertheless, any of these two copies would actually provide us with an irreducible representation of the model as far as we do not allow time reversal operations.

On the other hand, although we have considered the invariance of the Gowdy geometries under the transformations generated by the diffeomorphism constraints, we have in fact not discussed the possible symmetries under global diffeomorphisms that cannot be connected with the identity transformation. Diffeomorphisms of this kind which are compatible with the form of the metric (4.26) are given, e.g., by a change of orientation in one of the angular coordinates, or by an interchange of the coordinates that correspond to Killing fields of the spacetime, i.e., $\omega$ and $\nu$. In this sense, the point of view that we have adopted is that the coordinates $\omega$ and $\nu$ are physically distinguishable and the orientation of all spatial coordinates fixed once and for all.

During the completion of this paper, we have become aware of an independent work by Ashtekar and Pierri [23], who also study the quantization of the family of Gowdy universes with the spatial topology of a three-torus. In that work, the discussion has nonetheless been restricted to the case in which the two commuting Killing fields of the model are hypersurface orthogonal.

\section{ACKNOWLEDGMENTS}

The author is grateful to P. F. González Díaz and N. Manojlovic for helpful conversations. He is also greatly thankful to A. Ashtekar for valuable comments and discussions.
[1] A. Ashtekar, Phys. Rev. Lett. 57, 2244 (1986); Phys. Rev. D 36, 1587 (1987).

[2] A. Ashtekar, Lectures on Non-Perturbative Canonical Gravity, edited by L. Z. Fang and R. Ruffini (World Scientific, Singapore, 1991).

[3] A. Ashtekar, R. S. Tate, and C. Uggla, Int. J. Mod. Phys. D 2, 15 (1993); C. Barceló, L. J. Garay, P. F. González-Díaz, and G. A. Mena Marugán, Phys. Rev. D 53, 3162 (1996); H. Kastrup and T. Thiemann, Nucl. Phys. B399, 211 (1993); B436, 681 (1995); H. Kodama, Prog. Theor. Phys. 80, 1024 (1988); Phys. Rev. D 42, 2548 (1990); N. Manojlović and G. A. Mena Marugán, ibid. 48, 3704 (1993); Int. J. Mod. Phys. D 4, 749 (1995); G. A. Mena Marugán, Class. Quantum Grav. 11, 589 (1994); 11, 2205 (1994); Phys. Rev. D 50, 3923 (1994); 53, 3156 (1996).

[4] A. Ashtekar and M. Pierri, J. Math. Phys. (N.Y.) 37, 6250 (1996).

[5] A. Einstein and N. Rosen, J. Franklin Inst. 223, 43 (1937).

[6] K. Kuchar, Phys. Rev. D 4, 955 (1971).

[7] M. Allen, Class. Quantum Grav. 4, 149 (1987).

[8] E. Verdaguer, Phys. Rep. 229, 1 (1993).

[9] R. Geroch, J. Math. Phys. (N.Y.) 12, 918 (1971); 13, 394 (1972).

[10] D. Korotkin and H. Samtleben, "Poisson Realization and Quantization of the Geroch Group,' Report No. DESY/96245, gr-qc/9611061, 1996 (unpublished).
[11] V. A. Belinskii and V. E. Zakharov, Sov. Phys. JETP 48, 985 (1978); C. M. Cosgrove, J. Math. Phys. (N.Y.) 21, 2417 (1980); 22, 2624 (1981); 23, 615 (1982); W. Kinnersley and D. M. Chitre, ibid. 18, 1538 (1977); 19, 1926 (1978); 19, 2037 (1978); B. K. Harrison, Phys. Rev. Lett. 41, 1197 (1978); Phys. Rev. D 21, 1695 (1980); I. Hauser and F. J. Ernst, ibid. 20, 362 (1979); J. Math. Phys. (N.Y.) 21, 1126 (1980); 22, 1051 (1981); G. Neugebauer, J. Phys. A 12, L67 (1979).

[12] R. H. Gowdy, Ann. Phys. (N.Y.) 83, 203 (1974).

[13] V. Husain and L. Smolin, Nucl. Phys. B327, 205 (1989).

[14] B. K. Berger, Ann. Phys. (N.Y.) 83, 458 (1974); 156, 155 (1984).

[15] V. Husain, Phys. Rev. D 50, 6207 (1994).

[16] V. Husain, Phys. Rev. D 53, 4327 (1996).

[17] A. Ashtekar, J. D. Romano, and R. S. Tate, Phys. Rev. D 40, 2572 (1989).

[18] R. M. Wald, General Relativity (University of Chicago Press, Chicago, 1984).

[19] G. A. Mena Marugán, Int. J. Mod. Phys. D 3, 513 (1994).

[20] S. Mizoguchi, Phys. Rev. D 51, 6788 (1995).

[21] A. Galindo and P. Pascual, Mecánica Cuántica (Alhambra, Madrid, 1978).

[22] See, for instance, M. Reed and B. Simon, Methods of Modern Mathematical Physics, II: Fourier Analysis, Self-Adjointness (Academic, New York, 1975).

[23] A. Ashtekar and M. Pierri (in preparation). 